\title{
Stripe formation: A quantum critical point for cuprate superconductors
}

\author{
C. Castellani, C. Di Castro, and M. Grilli \\ Istituto di Fisica della Materia e Dipartimento di Fisica, Università di Roma "La Sapienza", \\ Piazzale A. Moro 2, 00185 Roma, Italy
}

\begin{abstract}
We discuss the effects of a quantum critical point located nearby optimum doping and related to local charge segregation (stripe phase). The fluctuations in the critical region produce at the same time a strong pairing mechanism and a non-Fermi liquid behavior in the normal phase above the superconducting critical temperature. Superconductivity is a stabilizing mechanism against charge ordering, i.e. the incommensurate charge density wave quantum critical point is unstable with respect to superconductivity. A complete scenario for the cuprates is presented.
\end{abstract}

\section{INTRODUCTION}

The presence of a critical point at zero temperature (Quantum Critical Point, QCP) ruling the physical properties of the superconducting cuprates was repeatedly suggested [1] 6]: In the quantum critical region above the QCP no energy scale besides temperature controls the physics and strong critical fluctuations can mediate singular interactions between quasiparticles, providing both a strong pairing mechanism and a source of non-Fermi liquid (non-FL) behavior in the normal-state 何.

A natural candidate where to place the QCP is the region nearby optimum doping where the highest critical temperatures and the best non-FL metal appear. Indeed the existence of a QCP near optimum doping is supported by several experimental findings. In particular recent transport measurements in $\mathrm{La}_{2-\mathrm{x}} \mathrm{Sr}_{\mathrm{x}} \mathrm{CuO}_{4}$ (LSCO) investigated the normal phase of these systems when superconductivity is suppressed under strong magnetic field [7. This analysis shows the existence of a QCP near optimum doping. Evidences in this sense are also provided by neutron scattering [8], by many experiments showing qualitative changes of behavior taking place near optimum doping between underdoped and overdoped samples and by the fact that several quantities (resistivity, Hall number, uniform susceptibility) show a scaling behavior with a typical energy scale, which vanishes at optimum doping [9].

Many indications exist that the above QCP involves charge ordering. Generically, it seems quite likely that charge degrees of freedom play a major role, since the disordered region of this QCP coincides with the highly metallic overdoped regime. Experimentally a direct observation of charge-driven ordering was possible by neutron scattering 116], in $\mathrm{La}_{1.4} \mathrm{Nd}_{0.4} \mathrm{Sr}_{0.12} \mathrm{CuO}_{4}$ where the related Bragg peaks were detected. For this specific compound the low-temperature-tetragonal lattice structure pins the charge-density waves (CDW) and gives static order and semiconducting behavior (see also the case of $\left.\mathrm{La}_{1.88} \mathrm{Ba}_{0.12} \mathrm{CuO}_{4}\right)$. Increasing the $\mathrm{Sr}$ content at fixed $\mathrm{Nd}$ concentration, the pinning effect is reduced lead- ing to metallic and superconducting behavior. In this latter case, the existence of dynamical incommensurate CDW (ICDW) fluctuations is suggested by the presence of incommensurate dynamic spin scattering, although the charge peaks are too weak to be observed. In this regard, also $\mathrm{La}_{2-\mathrm{x}} \mathrm{Sr}_{\mathrm{x}} \mathrm{CuO}_{4}$ is expected to display dynamical charge fluctuations with a doping-dependent spatial modulation as observed in the magnetic scattering [17]. ICDW have also been inferred from extended X-ray absorption fine structure (EXAFS) experiments both in optimally doped LSCO [18] and $\mathrm{Bi}_{2} \mathrm{Sr}_{2} \mathrm{CaCu}_{2} \mathrm{O}_{8+\mathrm{x}}$ (Bi2212) [19].

The above phenomenology supports the scenario of a proximity to an ICDW transition [4] 6], which in the absence of superconductivity would be located at zero temperature near the optimum doping. Large superconducting critical temperatures are most naturally expected where strong attractive critical fluctuations occur. Then, superconductivity takes place and the CDW instability is hindered. The CDW instability can only show its effects above $T_{c}$ as responsible in the quantum critical region for the best non-FL behavior occurring near optimum doping in all classes of cuprates. The presence of an ICDW-QCP is not alternative to the existence of an AF-QCP and the two QCP's control the behavior of the system at different dopings. It is worth noting that within the ICDW scenario, the ICDW stripes constitute the substrate to sustain the antiferromagnetic (AF) fluctuations far away from the AF-QCP. In the underdoped compounds, the ICDW instability would occur at finite temperature, were it not for the quenching due to superconducting local fluctuations which can give rise to the appearance of charge and spin gaps, as experimentally found [10 15] at a temperature $T^{*}$ above $T_{c}$. According to this proposal, the underlying hindered charge instability provides a strongly temperature dependent pairing potential [6]. This explains the high crossover temperature $T^{*}$ of the ( $d$-wave) pair formation and the peculiar doping dependence of $T^{*}$, which strongly increases with decreasing doping, [10 12] while the superconducting gap at $\mathrm{T}=0$ remains nearly constant [11, 15 . 


\section{THE ICDW INSTABILITY: THE FORMATION MECHANISM}

The occurrence of an ICDW-QCP is theoretically substantiated by considering the interplay between electronic phase separation (PS) and long-range Coulombic (LRC) repulsion.

PS is a common feature of strongly correlated electron systems: It has been found in magnetic models 20 23, in models with nearest-neighbour Coulomb interactions 24 29, and in models with phononic interactions 30 33. Indeed a strong on-site correlation drastically renormalizes the kinetic energy, which would tend to delocalize the carriers. Then short-range interactions (magnetic, phononic, nearest-neighbor coulombic...) introducing effective attractions between the carriers may dominate and give rise to charge aggregation in highly doped metallic regions together with charge depletion in spatially separated (magnetically ordered) regions with no itinerant charges. As it was first noticed in [22,25], pair formation always occur before and nearby PS. Therefore "phase separation does not spoil superconductivity. Pair formation at fixed doping is rather a stabilizing mechanism with respect to phase separation between the normal phases" 22.

It was then pointed out 34 that LR Coulomb forces effectively oppose the separation of charged particles suppressing long-wavelenght density fluctuations. This may lead to either dynamical slow density fluctuations 34 or ICDW 26, 4, 35. In this latter case microscopic calculations in the limit of strong electron-electron interaction were carried out both in the single-band infinite- $\mathrm{U}$ Hubbard model in the presence of an Holstein electronphonon coupling (Hubbard-Holstein model) [4] 31] and in the infinite-U three-band Hubbard model extended with a nearest-neighbor $(\mathrm{Cu}-\mathrm{O})$ repulsion $\mathrm{V}$ 36]. Similar results were found in both models thus confirming that the interplay between PS and LRC forces provide a robust mechanism for charge ordering instabilities. Despite the different dynamics, in both cases a similar singular attraction arises of the form

$$
\Gamma(\mathbf{q}, \omega) \approx \tilde{U}-\frac{1}{4} \sum_{\alpha} \frac{V}{\kappa^{2}+\omega_{\mathbf{q}}^{\alpha}-i \gamma \omega}
$$

where $\tilde{U}$ is the residual repulsive interaction between the quasiparticles and $\gamma$ is a damping parameter. The sum is over the four equivalent vectors of the CDW instability $\mathbf{q}^{\alpha}=\left( \pm q_{c}, 0\right),\left(0, \pm q_{c}\right)$ and $\omega_{\mathbf{q}}^{\alpha}=2\left(2-\cos \left(q_{x}-q_{x}^{\alpha}\right)-\right.$ $\left.\cos \left(q_{y}-q_{y}^{\alpha}\right)\right)$. This expression is used to reproduce the behavior $\sim-1 /\left(\kappa^{2}+\left(q_{x}-q_{x}^{\alpha}\right)^{2}+\left(q_{y}-q_{y}^{\alpha}\right)^{2}\right)$ for $q \rightarrow q^{\alpha}$ while mantaining the lattice periodicity.

The mass term $\kappa^{2}=a\left(\delta-\delta_{c}\right)$ is found [4] to be linear in the doping deviation from the critical concentration. For reasonable band-structure parameters the instability first occurs at $\delta_{c} \approx 0.2$ with $q_{c} \lesssim 1$. From our analysis the rather large density of states near the $( \pm \pi, 0)$ and $(0, \pm \pi)$ points tends to favor instabilities at or close to the $(1,0)$ or $(0,1)$ directions. However, due to the rather small values of $q_{c}$, the scattering is quite strong, although non-singular, in all directions for $|\boldsymbol{q}| \approx\left|\boldsymbol{q}_{c}\right|$.

\section{THE ICDW-QCP IN THE ABSENCE OF PAIRING}

In the light of the generality of the results of the previous section, we now discuss the properties of the ICDWQCP without a specific reference to a microscopic model, thus pointing out the robust generic features of the scenario. For the sake of definiteness we will assume the form (11) to be generically valid and we will also assume a simple Gaussian behavior of the QCP.

Around a QCP three regions are usually identified (thin solid lines in Fig. 1).

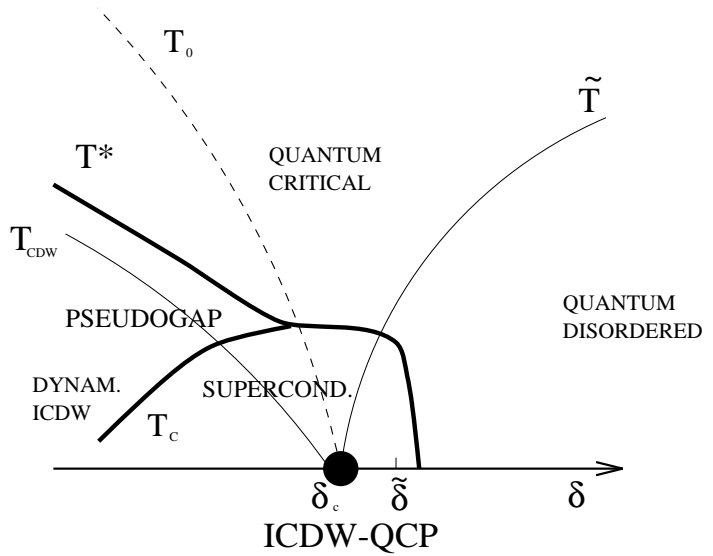

FIG. 1: Schematic structure of the temperature vs. doping $\delta$ phase diagram around the ICDW-QCP. On the right, $T<\tilde{T}$ : Quantum disordered region $\left[\xi^{-2} \approx\left(\delta-\delta_{c}\right)\right]$; In the middle, $T>T_{C D W}, \tilde{T}$ : Quantum critical (classical gaussian) region $\left[\xi^{-2} \approx T\right]$; On the left, $T<T_{C D W}$ : "ordered" ICDW phase. The heavy line indicates the region of local (pseudogap) or coherent (superconducting) pairing. In the presence of pairing, i.e. for $T<T^{*}$, the ICDW "order" is purely dynamical.

The quantum disordered (QD) regime on the right would in the present case correspond to the overdoped region, where $\kappa^{2}=\xi^{-2} \sim a\left(\delta-\delta_{c}\right)^{2 \nu}$. Within the adopted classical gaussian approximation, we take $\nu=1 / 2$. Increasing the temperature one enters in the so-called classical gaussian regime (quantum critical, QC), where $\kappa^{2}$ becomes a function of $T, \kappa^{2} \sim b T^{(d+z-2) / z}$, where $d$ is the spatial dimension and $z$ is the dynamical critical index. The proper $z$ is $z=2$ for CDW as one sees from the fluctuation propagator (11). $a$ and $b$ are model-dependent positive constants. $\tilde{T}$ marks the crossover between the two regimes. 
The region on the left of the QCP would generically correspond to the ordered ICDW phase occurring below a critical temperature $T_{C D W}(\delta)$ starting from the QCP at $\delta_{c}(T=0)$. The true critical line is depressed with respect to its mean-field expression (sketched by the dashed line $T_{0}$ of Fig. 1). The region between the two curves $T_{0}$ and $T_{C D W}$ is dominated by strong precursor effects.

The quantum disordered region on the right corresponds to the overdoped region of the cuprates, where a crossover to a low-temperature FL takes place. On other hand the classical gaussian region around optimum doping is characterized by the absence of energy scales, but the temperature. Here the best non-FL behavior is obtained. In particular, with the scattering of the form given in Eq. (1), a linear-in-T resistivity is expected in $d=2$, while for $d=3, \rho(T) \sim T^{3 / 2}$. For magnetically mediated scattering, in Ref. [37] the objection was raised that only few "hot" points would feel strong scattering contributing to the above behavior. Generically, all other points would contribute to the lower $T^{2}$ behavior. However, for ICDW, the fact that typical $q_{c}$ are fairly small and the strong isotropic character of $\Gamma(q)$ make this objection less relevant.

It is worth noting that the quantum critical behavior in transport may extend to the overdoped region down to low temperatures well below $\tilde{T}$. Indeed, whereas the critical crossover line $\tilde{T}(\delta)$ is established by matching the behavior of $\kappa^{2}$ as a function of $\left(\delta-\delta_{c}\right)$ and $T$ in the QD and QC regions respectively, transport crucially involves temperature, i.e. frequency, scales. Then, the temperature below which the fluctuations feel the presence of the mass $\kappa^{2}$ is determined by the condition $\gamma \omega \sim \gamma T \sim \kappa^{2}$. Clearly for large enough $\gamma$ quite low temperatures $T_{F L}$ can be reached before the singular interaction is cut off leading to FL behavior $\left(\rho \propto T^{2}\right)$.

Within our scenario the temperature $T_{0}$ marks the onset of the ICDW precursors and is characterized by a loss of spectral weight at low energies giving rise to a uniform decrease of the density of states near the Fermi energy. This would show up as the well known decrease of the uniform magnetic susceptibility below a characteristic temperature, which vanishes by approaching from below the optimum doping [39]. In underdoped $\mathrm{YBa}_{2} \mathrm{Cu}_{3} \mathrm{O}_{6+\mathrm{x}}$ (YBCO) compounds, this last temperature has also been put in correspondence 40,38 with the temperature below which the planar resistivity $\rho_{a b}$ deviates from its linear behavior.

The scenario presented so far should find a wider physical correspondence whenever the superconducting pair formation is forbidden. Boebinger et al. |7] report the results of transport experiments in LSCO, in the presence of high magnetic fields, allowing to access the normal phase underlying the superconducting region. They assess the presence of a metal-insulator transition in the underdoped region ending near optimum doping $(x \approx 0.17)$ at $T=0$. Actually, their work not only shows the exis- tence of $\mathrm{QCP}$ different from the $\mathrm{AF}-\mathrm{QCP}$, but also provides a clear evidence that the nature of this instability is related to charge-ordering.

The experimental line separating the planar metal from the insulator would correspond in our picture to the "true" $T_{C D W}$ critical temperature as a function of doping, which manifests itself when superconductivity is suppressed. We also observe that the resistivity curve for $x=0.12$, i.e. near the "magic" doping $1 / 8$, displays an insulating behavior at a much higher temperature than for values of $x$ immediately nearby. This shows that commensurability indeed plays a relevant role in establishing the insulating phase, thereby indicating that spatial order of the charge degrees of freedom should be involved also away from commensurability. This would also agree with the observation made in Ref. [7] that the system is rather clean $\left(k_{F} l \sim 13\right)$ and the disorder by itself cannot be the source of the transition 41]

Concerning the location of the QCP, we would expect that both the metal-insulator transition line (which we identified with $T_{C D W}$ ) and the temperature of the onset of pseudogap (identified by $T_{0}$ ), would end to the same point at $T=0$. Fig. 3 of Ref. [7] seem to assign a value of $x_{c}=0.17$ to the critical doping $\delta_{c}$. On the other hand from Fig. 8 of Ref. [38] reporting the temperature $T_{0}$ for many physical quantities, one would deduce a somewhat larger value $x_{c} \gtrsim 0.2$ (in Ref. [38] our $T_{0}$ is indicated by $\left.T^{*}\right)$. However, this discrepancy can be solved by noting that the resistivity curve at $x=0.17$ in Fig. 1 of Ref. [7] is likely still affected by superconductivity at the lowest temperature, and the metal-insulator transition at this filling seems to occur at a still finite temperature (of about $15 \mathrm{~K}$ ). Therefore, the published data of Fig. 1(b) in Ref. [7] are compatible with a shift of the metalinsulator transition at $T=0$, towards dopings that are larger than the assigned value.

An additional observation can be made by contrasting the behavior of LSCO systems with La-doped $\mathrm{Bi}_{2} \mathrm{Sr}_{2} \mathrm{CuO}_{\mathrm{y}}$ (Bi-2201) materials 42]. These latter systems are slightly overdoped. Therefore it is not surprising that the low temperature behavior of the planar resistivity is always metallic in Bi-2201. On the other hand they are much more anisotropic than the LSCO systems. Therefore it is again natural to find that $\rho_{c}$ is always semiconducting in Bi-2201. This strongly twodimensional character also accounts for the robust linear behavior of the resisitivity which is expected in the quantumcritical region above a twodimensional QCP. On the contrary, the transverse hopping, being larger in LSCO, easily becomes coherent by increasing doping, giving rise to threedimensional metallic behavior. In this case the observed $T^{3 / 2}$ behavior for the planar resistivity in the overdoped regime is explained as the result of the quantum critical behavior in three dimension [43]. 


\section{THE ICDW-QCP IN THE PRESENCE OF PAIRING}

The singular interaction of Eq.(11), when considered in the particle-particle channel, provides a strong pairing mechanism in the proximity of the critical point. By allowing for superconducting pairing the thick solid lines in Fig. 1 schematically represent the crossover $\left(T^{*}\right)$ to local pairing or the coherent-superconducting transition $\left(T_{c}\right)$ in the phase diagram near the ICDW-QCP, thus eliminating the $T_{C D W}$ and part of the $\tilde{T}$ line.

The most apparent and generic result is that pairing has $d$-wave symmetry [5] and, being mediated by an interaction rapidly varying with $\kappa^{2}$ [cf. Eq.(1)], strongly depends on temperature or doping. Roughly, the $d$-wave becomes favorable since the average repulsion felt by the $s$-wave paired electrons exceeds the loss in condensation energy due to the vanishing of the order parameter along the nodal regions. Among the $d$ waves, the $d_{x^{2}-y^{2}}$ is preferred because the nodes occur in regions with small density of states.

The superconducting critical temperature evaluated in the BCS approach [44] shows a strong increase upon decreasing $\kappa^{2}$. The actual behavior of $T_{c}$ is then obtained by introducing the doping and temperature dependence of $\kappa^{2} \equiv \kappa^{2}\left(\delta-\delta_{c}, T\right)$. An additional (less important) doping dependence is due to the variation of the chemical potential with respect to the van Hove singularity (VHS). In the quantum disordered phase the pairing potential becomes stronger and stronger by decreasing doping towards $\delta_{c}$ and a rapid increase in $T_{c}\left[\simeq T_{c}\left(\kappa^{2}\left(\delta-\delta_{c}, T=\right.\right.\right.$ $0)$ )] will result. At a given doping $\tilde{\delta} \gtrsim \delta_{c}$ the BCS superconducting temperature will reach the crossover line $\tilde{T}$ separating the quantum disordered from the classical gaussian region. In this latter region $\kappa^{2}$ weakly depends on doping and a plateau in $T_{c}$ is reached.

In this scheme the maximal critical temperature is obtained near the quantum-disordered/quantum-critical crossover $\left(\delta_{\text {opt }} \approx \tilde{\delta}\right)$. Of course this is only an estimate depending on the use of a weak coupling BCS scheme and of a model dependent evaluation of $\kappa^{2}\left(\delta-\delta_{c}, T\right)$. Nevertheless, we remark that the rapid variation of $T_{c}$ with doping in the overdoped region, observed in many cuprates, and the plateau near optimum doping are naturally captured by our description.

Notice that in discussing $T_{c}$ vs doping we have used the fact that the main doping dependence is via $\kappa^{2}$. Strong variations of $T_{c}$ with doping are hardly obtained in terms of a dependence on band parameters only (specifically, tuning the VHS). They are instead quite natural in the context of proximity to an instability, where doping controls the effective potential itself and not only the density of states. This agrees with the experimental finding that at the maximum $T_{c}$ the VHS is not at the Fermi energy but below it 45].
The region on the left of the mean-field critical curve for the ICDW transition is characterized by strong thermal fluctuations leading to ICDW precursors. The ICDW fluctuations in the underdoped region become critical in the proximity of the line $T_{C D W}(\delta)$ where the ICDW transition would occur in the absence of superconducting pairing. Approaching $T_{C D W}$ the attractive fluctuations would lead to the formation of (local) pairs at the curve $T^{*}(\delta)$. As a consequence of strongly paired quasiparticles, below $T^{*}(\delta)$ pseudogap effects will show up as seen in many experiments (NMR, ARPES, optical conductivity, specific heat, ...). However, despite the strong pairing, the true superconducting critical temperature is lower than $T^{*}$ and it decreases inside the underdoped psudogap region. This occurrence is schematically depicted in Fig. 3 by the bifurcation of the heavy line.

Experimentally it is observed 15, 11, 12, that the lowtemperature gap in the underdoped cuprates weakly depends on doping, while $T^{*}$ increases fast by decreasing $\delta$. Although a full theory of this complex phenomenon is far from being available, we believe that this peculiar behavior requires a remarkable temperature dependence of the pairing potential as implied by our ICDW scenario in the underdoped region and cannot easily be accounted for by simple models of local pairing. In the underdoped region $\kappa^{2}$ in Eq. (11) has a modified temperature dependence given by the distance from the critical line $T_{C D W}$ Since the pair formation has a stabilizing effect on the ICDW instability, we introduce $\Delta_{M a x}$, the maximum value of $\Delta(\boldsymbol{k})$ in $k$-space as an additional cut-off in Eq. (1) by assuming $\kappa^{2} \equiv \operatorname{Max}\left[c\left|\Delta_{M a x}(T)\right|, c^{\prime}\left(T-T_{C D W}\right)\right]$. Despite the oversimplified form of $\kappa^{2}$, the $T$ behavior of $\Delta_{\text {Max }}$ bears a striking resemblance with the analogous quantity recently measured with ARPES in underdoped Bi-2212 samples [1], with a long tail extending up to large temperatures $T \approx T_{C D W} \approx T^{*}[6]$.

Of course the above BCS treatment only deals with the amplitude of the gap and says nothing on the way a true superconducting phase coherence is established below a critical temperature $T_{c}<T^{*}$. For this we have to invoke phase fluctuations as in the usual (large) negative$\mathrm{U}$ Hubbard model. Here the additional features (the peaked $q$-dependence of the pairing potential, and precursors of stripe formation) are expected to enhance the role of phase fluctuations.

It is also important to emphasize once again that the occurrence of local pairing prevents the actual establishing of the ICDW long-range order 46], so that $T_{C D W}$ and the ICDW-QCP loose their meaning of a true transition , and they merely indicate the crossover regions where pairing and strong ICDW (dynamical) order selfconsistently interplay.

Acknowledgments: Part of this work was carried out with the financial support of the Istituto Nazionale di Fisica della Materia, Progetto di Ricerca Avanzata 1996. 
[1] S. Sachdev and J. Ye, Phys. Rev. Lett. 69, 2411 (1992); A. V. Chubukov, S. Sachdev, and J. Ye, Phys. Rev. B 49, 11919 (1994).

[2] A. Sokol and D. Pines, Phys. Rev. Lett. 71, 2813 (1993); P. Monthoux and D. Pines, Phys. Rev. B 47, 6069 (1993), and references therein.

[3] C. M. Varma, Phys. Rev. Lett. 75, 898 (1995); C. M. Varma, preprint, cond-mat 9607105 and references therein.

[4] C. Castellani, C. Di Castro, and M. Grilli, Phys. Rev. Lett. 75, 4650 (1995)

[5] A. Perali, C. Castellani, C. Di Castro and M. Grilli, Phys. Rev. B 54, 16216 (1996)

[6] (a) C. Castellani, C. Di Castro, and M. Grilli, Z. Phys. B 103, 137 (1997); (b) C. Castellani, C. Di Castro, and M. Grilli, to appear in Physica C.

[7] G. S. Boebinger, et al., Phys. Rev. Lett. 77, 5417 (1996).

[8] T. E. Mason, et al., Phys. Rev. Lett. 77, 1604 (1996).

[9] See the discussion and Refs. 29-42 in Ref. [6](a).

[10] D. S. Marshall, et al., Phys. Rev. Lett. bf 76, 4841 (1996).

[11] J. M. Harris, et al., preprint 1996, cond-mat 9611010.

[12] H. Ding, et al., Nature 382, 51 (1996).

[13] J. Rossat-Mignod, et al., Physica C 185-189.

[14] For a recent review see C. Berthier et al., J. de Physique I 6, 2205 (1996) and references therein.

[15] J. W. Loram, et al., Phys. Rev. Lett. 71, 1740 (1993); J. W. Loram, et al. Physica C 235-240, 134 (1994).

[16] J.M. Tranquada, B.J. Sternlieb, J.D. Axe, Y. Nakamura, and S. Uchida, Nature 375, 561 (1995).

[17] K. Yamada, et al. preprint 1996.

[18] A. Bianconi, et al., Phys. Rev. Lett. 76, 3412 (1996).

[19] A. Bianconi, et al., Phys. Rev. B 54, 12018 (1996) and references therein.

[20] V. J. Emery, S. A. Kivelson and H. Q. Lin, Phys. Rev. Lett. 64, 475 (1990)

[21] M. Marder, N. Papanicolau and G. C. Psaltakis, Phys. Rev. B 41, 6920 (1990)

[22] N. Cancrini, S. Caprara, C. Castellani, C. Di Castro, M. Grilli and R. Raimondi, Europhys. Lett. 14, 597 (1991).

[23] E. Dagotto, Rev. Mod. Phys. 66, 763 (1994).
[24] Y. Bang, G. Kotliar, C. Castellani, M. Grilli, and R. Raimondi, Phys. Rev. B 43, 13724 (1991).

[25] M. Grilli, R. Raimondi, C. Castellani, C. Di Castro, and G. Kotliar, Phys. Rev. Lett. 67, 259 (1991)

[26] R. Raimondi, C. Castellani, M. Grilli, Y. Bang, and G. Kotliar, Phys. Rev. B 47, 3331 (1993)

[27] F. Bucci, C. Castellani, C. Di Castro, and M. Grilli, Phys. Rev. B 52, 6880 (1995).

[28] J. C. Hicks, A. E. Ruckenstein, and S. Schmitt-Rink, Phys. Rev. B 45, 8185 (1992).

[29] M. E. Simon and A. A. Aligia, Phys. Rev. B 53, 15327 (1996).

[30] M. Grilli and C. Castellani, Phys. Rev. B 50, 16880 (1994).

[31] F. Becca, M. Tarquini, M. Grilli, and C. Di Castro, Phys. Rev. B 54, 12443 (1996).

[32] R. S. Markiewicz, J. Phys. Cond. Matt. 2, 665 (1990).

[33] R. S. Markiewicz, J. Phys. Chem. Sol. 58, 1179 (1997) and references therein.

[34] V. J. Emery and S. A. Kivelson, Physica C 209, 597 (1993).

[35] U. Low, V. J. Emery, K. Fabricius, and S. A. Kivelson, Phys. Rev. Lett. 72, 1918 (1994)

[36] F. Becca, F. Bucci, and M. Grilli, cond-mat 9707109.

[37] R. Hlubina and T. M. Rice, Phys. Rev. B 51, 9253 (1995).

[38] B. Batlogg, et al., Physica C 235-240, 130 (1994).

[39] D. C. Johnston, Phys. Rev. Lett. 62, 957 (1989).

[40] T. Ito, et al., Phys. Rev. Lett. 70, 3995 (1993).

[41] However, disorder can play an important role in modifying $T_{C D W}$ and in determining the $\log T$ behavior of the resistivity in the insulating CDW phase.

[42] Y. Ando, et al., Phys. Rev. Lett. 77, 2065 (1996).

[43] T. Moriya, et al., Physica C 185-189, 114 (1991).

[44] It should, however, be kept in mind that even in the uniform (i.e. non-ordered CDW), the evaluation of a quantitatively reliable superconducting critical temperature is a difficult task, since the pairing is mediated by singular interactions.

[45] Z. X. Shen and D. S. Dessau, Phys. Rep. 253, 1 (1995).

[46] Indeed it was shown in a Kondo-lattice-like model that superconductivity stabilizes phase separation already at mean field level 22. 